\title{
Tumor neuroendocrino de la válvula ileocecal con metástasis hepática única. Presentación de caso
}

\section{A case of a neuroendocrine tumor of the ileocecal valve with a single hepatic metastasis}

Pedro Rosales Torres, MD, ${ }^{1}$ Rafael Pila Pérez, MD, ${ }^{1}$ Pedro León Acosta, MD, ${ }^{* *}$ Rafael Pila Peláez, MD. ${ }^{1}$

1 Hospital Provincial Docente "Manuel Ascunce Domenech". Camagüey. Cuba.

*Correspondencia: leonacostapedro@gmail.com.

Fecha recibido: $\quad 05-02-18$

Fecha aceptado: 03-04-18

\begin{abstract}
Resumen
Los tumores neuroendocrinos (TNE) del tracto gastrointestinal son muy infrecuentes, el intestino delgado y principalmente el íleon son los sitios donde ocurren con mayor frecuencia. Tienen la capacidad de secretar péptidos y aminas bioactivas. La Organización Mundial de la Salud (OMS) los clasifica de acuerdo con el grado de diferenciación y conducta biológica en 5 grados. Las manifestaciones clínicas pueden estar ausentes o ser inespecíficas. Se reporta el caso de un paciente de 49 años de edad quien fue ingresado por dolor abdominal en hemiabdomen derecho, acompañado de aerogastria, vómitos y síndrome constitucional; en el examen físico se encontró hepatomegalia dolorosa con nódulo único de $2,5 \mathrm{~cm}$ aproximadamente. La citología por aspiración con aguja fina (CAFF) y la laparoscopia con biopsia diagnosticaron una metástasis hepática única de un TNE de origen gastrointestinal, que se confirmó con la colonoscopia con ileoscopia encontrando un tumor subepitelial en la válvula ileocecal. La inmunohistoquímica realizada ayudó a la confirmación del diagnóstico. El tratamiento puede ser quirúrgico; sin embargo, este paciente rechazó esta terapia, por lo que se trató con quimioterapia y radioterapia. Lo fundamental en esta entidad es el diagnóstico precoz, que pocas veces se realiza por lo infrecuente de la enfermedad.
\end{abstract}

\section{Palabras clave}

Tumor neuroendocrino, clasificación, diagnóstico, colonoscopia.

\begin{abstract}
Neuroendocrine tumors of the gastrointestinal tract are very rare tumors that most frequently occur in the small intestine, primarily in the ileum. They secrete bioactive peptides and amines. The World Health Organization (WHO) classifies them into five grades according to the degree of differentiation and biological behavior. Clinical manifestations may be absent or nonspecific. We report the case of a 49-year-old patient who was admitted due to abdominal pain in the right hemiabdomen, accompanied by aerogastria, vomiting and constitutional syndrome. A painful hepatomegaly with a single nodule of approximately 2.5 $\mathrm{cm}$ was found during physical examination. CAFF and laparoscopy with biopsy diagnosed a single hepatic metastasis of a neuroendocrine tumor of gastrointestinal origin. This was confirmed by colonoscopy with ileoscopy which found a subepithelial tumor in the ileocecal valve. Immunohistochemistry helped confirm the diagnosis. Treatment for this type of tumor can be surgical, but this patient rejected surgery. It was treated with chemotherapy and radiation therapy. Early diagnosis of this entity is fundamental but is rare because of the infrequency of the disease.
\end{abstract}

Keywords

Neuroendocrine tumor, classification, diagnosis, colonoscopy. 


\section{INTRODUCCIÓN}

Los tumores neuroendocrinos del sistema gastrointestinal (TNE) constituyen una entidad clínico-patológica heterogénea muy agresiva, constituye menos del $5 \%$ de todas las neoplasias del aparato digestivo (1) y su incidencia varía de $2,5 \%$ a $5 \%$ casos $/ 100000(1,2)$. Se derivan de las células neuroendocrinas del aparato gastrointestinal, por lo que poseen capacidad neurosecretora; es decir, la capacidad de secretar péptidos y aminas bioactivas, siendo la principal la 5-hidroxitriptamina (5-HT) o serotonina (2). Se localizan con mayor frecuencia en el aparato gastrointestinal (74 \%) y en particular el intestino delgado, principalmente el íleon, donde se reporta con mayor frecuencia (30\%) como en este caso; sin embargo, también pueden encontrarse en el sistema broncopulmonar (25\%) (3). El restante $1 \%$ ocurre en la laringe, vesícula biliar, conductos biliares extrahepáticos, hígado, bazo y genitales masculinos y femeninos (4). Los TNE se denominan de acuerdo con la hormona que liberan y solo si esta se encuentra en cantidades medibles $y$ es funcionalmente activa; de lo contrario se denominan neuroendocrinos $(1,3)$. El objetivo de este trabajo es presentar el caso de un paciente con TNE de la válvula ileocecal, que resulta muy infrecuente en nuestro medio.

\section{CASO CLÍNICO}

Paciente de 49 años de edad, masculino, blanco, carpintero, fumador de una cajetilla de cigarrillos diaria desde hace 20 años y alcohólico crónico, bebedor de $1 / 2$ litro de ron diariamente durante los últimos 15 años, sin antecedentes familiares de interés. Refirió que en los últimos 6 meses aproximadamente comenzó con dolor abdominal difuso en todo el abdomen, especialmente en el hemiabdomen derecho; ha ido en aumento, era punzante y de inicio en el hipocondrio derecho. Luego, el dolor se intensificó, acompañado de náuseas, vómitos en número de 3 , aerogastria y distensión abdominal. Acudió a un facultativo quien le indicó un tratamiento, pero no mejoró y a la sintomatología anterior se añadió estreñimiento, astenia, anorexia y pérdida de $10 \mathrm{~kg}$. Todo este cuadro lo obligó a consultar a otro especialista, quien decidió su ingreso.

\section{Examen físico}

Afectación del estado general, mucosas húmedas y normocoloreadas, sin adenopatías en cadenas ganglionares periféricas, ni edemas, ni ictericia. El examen cardiorrespiratorio fue normal con tensión arterial (TA) 130/80 mm Hg. La frecuencia cardíaca central fue de 84 latidos por minuto (lpm).

$\mathrm{El}$ abdomen era difusamente doloroso en el hemiabdomen derecho con hepatomegalia que rebasó el reborde costal $2 \mathrm{~cm}$, se palpó una masa redondeada de aproximadamente $2 \mathrm{~cm}$, dolorosa y fija. A nivel de la fosa ilíaca derecha se detectó una zona indurada y ligeramente dolorosa. No se encontraron otras alteraciones. El resto del examen físico fue normal, incluida la esfera urológica y el fondo de ojo.

Los estudios analíticos, iones, enzimas, función renal, hepática y pancreática no mostraron alteraciones de importancia; solo una velocidad de sedimentación globular de 80 $\mathrm{mm} / \mathrm{1}^{\text {era }} \mathrm{h}$ (valor normal: hasta 20 ) y una lactato-deshidrogenasa (LDH) de $850 \mathrm{UI} / \mathrm{L}$ (valor normal: 130-300). La radiografía de tórax y el electrocardiograma (ECG) resultaron normales, y el ultrasonido abdominal mostró el hígado con nódulo único de $2,5 \mathrm{~cm}$, sin otras alteraciones.

Se realizó una citología por aspiración con aguja fina (CAAF), que informó positividad para células neoplásicas: tumor epitelial de bajo grado tipo adenocarcinoma de posible origen endocrino. Se realizó laparoscopia y biopsia por trocar grueso, que informó las mismas características que la CAAF (Figuras 1 y 2). La tomografía axial computarizada (TAC) de abdomen, retroperitoneo, tórax y mediastino resultaron sin anormalidades.

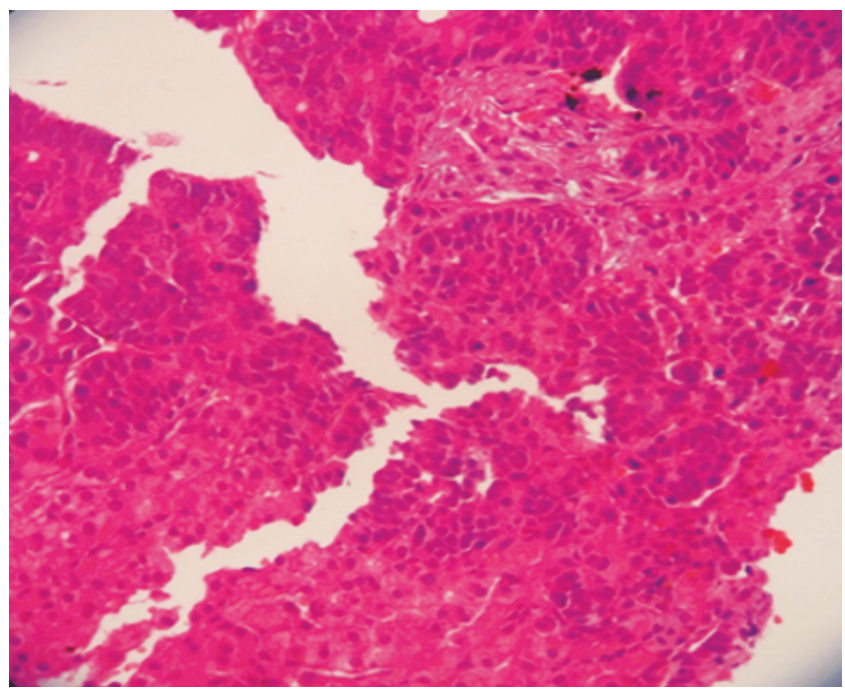

Figura 1. Microfotografía de biopsia hepática que muestra infiltración metastásica difusa por nidos celulares malignos. H/E-20 X.

El colon baritado (colon por enema) mostró defecto de lleno a nivel del íleon terminal.

La colonoscopia reveló una lesión de aspecto tumoral a nivel de la válvula ileocecal, redondeada de unos $4 \mathrm{~cm}$ de tamaño y del mismo color que la mucosa circundante; se apreció el signo de la tienda de campaña, que es la elevación de la pared intestinal con base ancha y vértice romo, sin pedículo con conservación de la mucosa intacta (Figuras 3 y 4). Se tomó una biopsia para el estudio histológico, que informó la presencia de un carcinoma neuroendocrino 


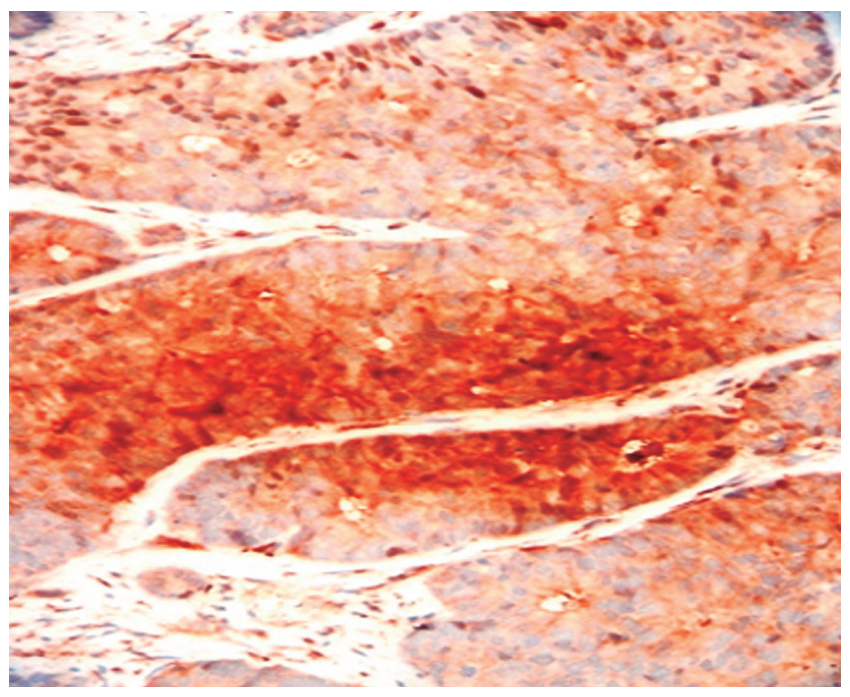

Figura 2. Marcaje intensamente positivo en zona tumoral con ENS. IHQ-20 X.

poco diferenciado grado III. La inmunohistoquímica fue positiva a enolasa neuroespecífica (NSE), sinaptofisina y Ki 67 (Figuras 5, 6 y 7 ).

El paciente se trasladó al Hospital Oncológico Madame Curie de la ciudad de Camagüey, donde se inició el tratamiento con quimioterapia y radioterapia.

\section{DISCUSIÓN}

No está clara la razón por la cual los tumores del intestino delgado tienen tan baja frecuencia; se ha propuesto que

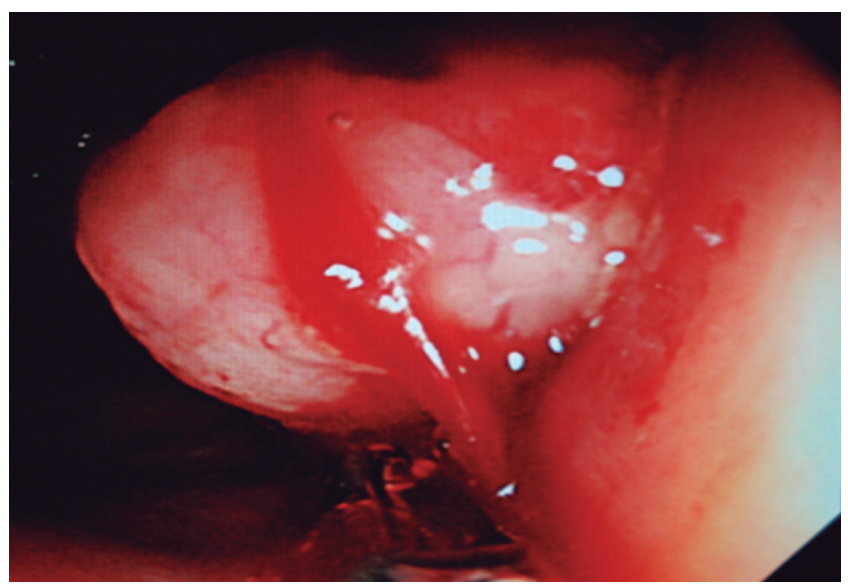

Figura 3. Imagen colonoscópica a nivel de la válvula ileocecal. Obsérvese la imagen tumoral exofítica. el alto contenido de líquidos causa menor irritación de la mucosa y una menor exposición a carcinógenos. Se ha sugerido que la mayor secreción de inmunoglobulina A (IgA) tiene un factor protector (5). Los TNE se originan del sistema endocrino gastrointestinal y se relacionan con los TNE de tiroides, páncreas y feocromocitomas, así como a las neoplasias endocrinas múltiples (MEN) en un $9 \%$ (2). El tracto gastrointestinal posee el mayor número de células neuroendocrinas del organismo, estas producen péptidos y aminas que regulan la motilidad y la digestión (5). Solo algunos ocasionan proliferación neoplásica, siendo esta menos frecuente a nivel de la submucosa del íleon terminal $(1,4)$ como en el paciente que presentamos.

Los TNE se denominan de acuerdo con las hormonas que producen. La clasificación de los TNE propuesta por Capella y adoptada por la OMS se basa en el sitio de origen, tamaño del tumor, extensión en el tejido circundante, angioinvasión, comportamiento biológico y diferenciación histológica en combinación con el estado funcionante o no funcionante $(1,2)$. Este paciente se encontraba en el grado III de esta clasificación. El pico de aparición es entre la sexta y séptima décadas de la vida; el $10 \%$ de estos tumores secreta mediadores bioactivos, lo que produce los síntomas típicos del síndrome carcinoide, pero por lo general consultan por dolor abdominal u obstrucción intestinal; sin embargo, el diagnóstico preoperatorio suele ser difícil por la evolución insidiosa de los síntomas (6). Estos tumores pueden permanecer asintomáticos durante años (generalmente antes de la aparición de metástasis) o presentar síntomas obstructivos, los cuales se hallan accidentalmente durante una cirugía o la realización de un estudio radiológico (5).

El diagnóstico de esta entidad debe incluir determinaciones de 5-HT en orinas de 24 horas para los carcinoides pro-

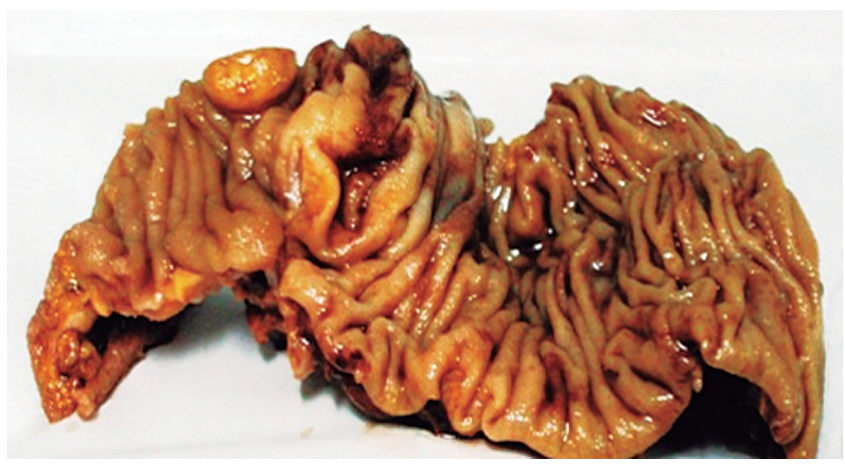

Figura 4. Macrofotografía a nivel del íleon terminal. Obsérvese la lesión tumoral redondeada de color amarillo oscuro a $1 \mathrm{~cm}$ y medio de la válvula ileocecal. 


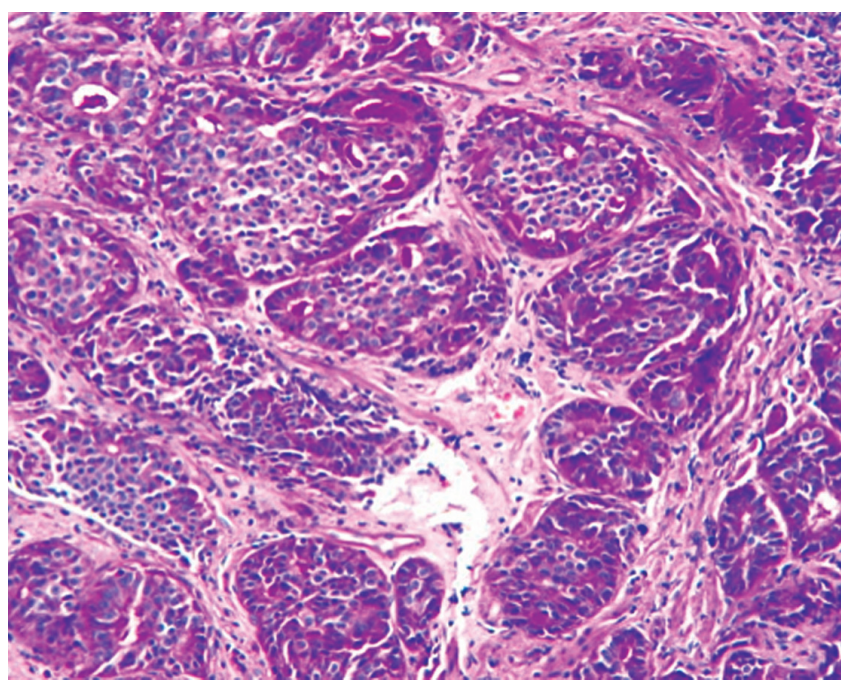

Figura 5. Microfotografía de la lesión intestinal que muestra nidos bien delimitados de células tumorales malignas con características histológicas neuroendocrinas. H/E-20 X.

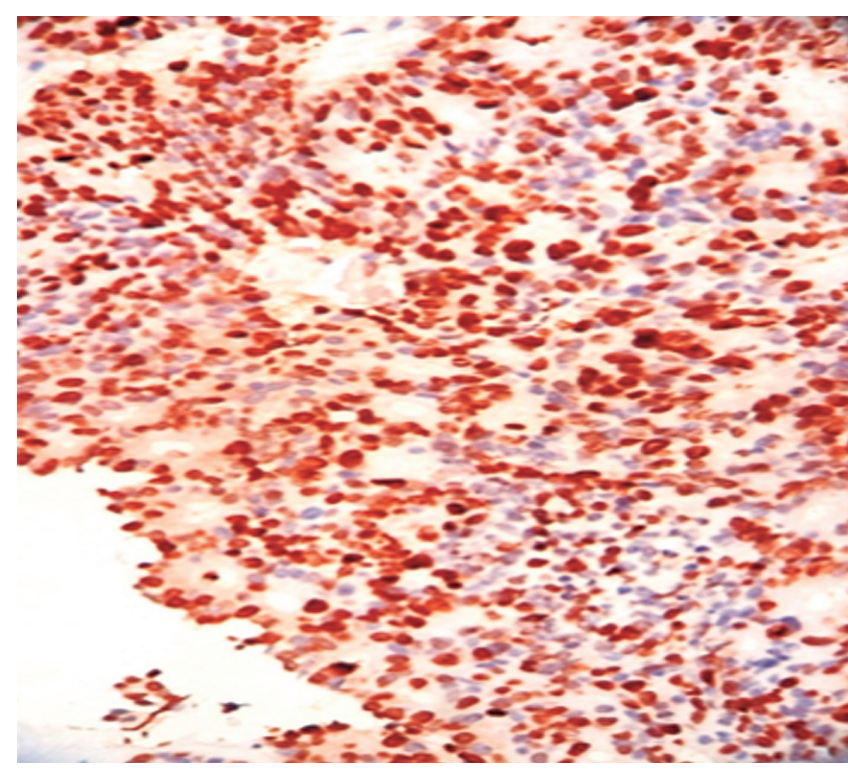

Figura 7. Lesión intestinal: positividad para Ki 67. IHQ-40 X.

ductores de serotonina, la cual no fue posible realizar por dificultades con el estudio. La TAC y resonancia magnética nuclear (RMN) han demostrado poseer similitud para el diagnóstico de metástasis regionales y a distancia con un $77 \%$ y un $80 \%$, respectivamente (7); en este paciente, la TAC con doble contraste fue muy efectiva; y fue igualmente útil la endoscopia digestiva, el tránsito intestinal y el ultrasonido abdominal. Para mejorar el diagnóstico de

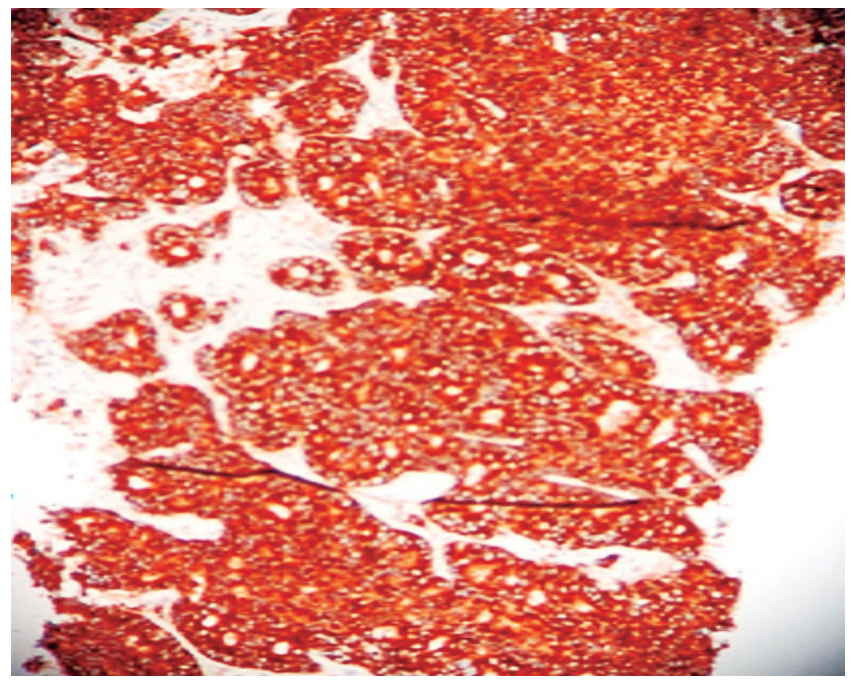

Figura 6. Lesión intestinal: reacción intensamente positiva para sinaptofisina. IHQ-20 X.

esta entidad se usa una serie de marcadores de inmunohistoquímica, entre ellos la ENS y los marcadores asociados con vesículas pequeñas (sinaptofisina). Hay una relación casi lineal entre el tamaño y la presencia de metástasis, el 85 $\%$ de los tumores de más de $2 \mathrm{~cm}$ produce metástasis (5), comprobado en este paciente.

El tratamiento para los TNE varía desde el tratamiento médico con agentes de quimioterapia y radioterapia, hasta el tratamiento quirúrgico con resección tumoral, dependiendo de la localización, el estadio en que se clasifique y los síntomas. En los pacientes con metástasis, la resección del tumor primario junto con los nódulos mesentéricos ha reportado mejoría notable de la sintomatología (8). La quimioterapia y radioterapia han demostrado poca utilidad en estos tumores (4). El pronóstico de los TNE del intestino delgado es desfavorable en comparación con los gástricos y los del recto (8). La tasa de supervivencia a los 10 años es del $65 \%$ al $73 \%$ para los tumores bien diferenciados y del $46 \%$ al $71 \%$ para la enfermedad localizada y a distancia $(2,3)$.

\section{CONCLUSIONES}

Los TNE del sistema gastrointestinal son entidades muy infrecuentes. Estos tumores tienen manifestaciones clínicas heterogéneas y pueden permanecer asintomáticos durante años. El diagnóstico diferencial incluye muchas causas de dolor abdominal. El tiempo entre el inicio de los síntomas y el diagnóstico es de 6 a 7 meses, siendo importantes la endoscopia digestiva ultrasonido y TAC de doble contrate. La terapia varía desde el tratamiento médico hasta 
el tratamiento quirúrgico, dependiendo de la localización, el estadio y los síntomas.

\section{Conflictos de intereses}

Los autores declaran no haber recibido ayuda económica o de otro tipo en la elaboración de este artículo.

\section{REFERENCIAS}

1. Alvarado Camello I. Tumores neuroendocrinos del aparato gastrointestinal y páncreas. Patología Rev latinoamericana. 2009;47(3):213-9.

2. Lawrence B, Gustafsson BI, Chan A, Svejda B, Kidd M, Modlin IM. The epidemiology of gastroenteropancreatic neuroendocrine tumors. Endocrinol Metab Clin North Am. 2011;40(1):1-18, vii. doi: 10.1016/j.ecl.2010.12.005.
3. Reynolds I, Healy P, Mcnamara DA. Malignant tumours of the small intestine. Surgeon. 2014;12(5):263-70. doi: 10.1016/j.surge.2014.02.003.

4. Pasieka JL. Carcinoid tumors. Surg Clin North Am. 2009;89(5):1123-37. doi: 10.1016/j.suc.2009.06.008.

5. Zúñiga D. Tumores neuroendocrinos gastrointestinales. Medicina legal de Costa Rica. 2013;30(1):89-98.

6. Gornas J, Varas M. Diagnóstico definitivo de los tumores neuroendocrinos mediante PAFF ecodirigidas por ultrasonido endoscópico. Rev Esp Enf Diag. 2011;103(3):123-8.

7. Mocellin S, Nitti D. Gastrointestinal carcinoid: epidemiological and survival evidence from a large population-based study (n = 25 531). Ann Oncol. 2013;24(12):3040-4. doi: 10.1093/annonc/mdt377.

8. Coan KE, Gray RJ, Schlinkert RT, Pockaj BA, Wasif N. Metastatic carcinoid tumors--are we making the cut? Am J Surg. 2013;205(6):642-6. doi: 10.1016/j.amjsurg.2012.05.036. 